CLINICAL CASE

\title{
MINIMAL INVASIVE AORTIC VALVE REPLACEMENT - CLINICAL STUDY
}

\author{
M. Robu', Aura Mazâlu', Celia Ciobanu', C. Voica', L. Dorobanțu', H.Moldovan ${ }^{1}$ \\ 'Cardiovascular Surgery Unit I, "Prof. Dr.C.C. Iliescu" The Emergency Institute for Cardiovascular Diseases \\ Corresponding author: Horațiu Moldovan \\ Phone no. 0040722301289 \\ E-mail: h_moldovan@hotmail.com
}

\begin{abstract}
The revolution of minimally invasive techniques from the last two decades lead to minimally invasive aortic valve replacement. Minimal " $J$ " shaped sternotomy extended in to the 4 intercostal space provides good access to the aortic root and the possibility to connect the patient to the extracorporeal circulation machine without the use of new instruments. We present a 69-year-old patient that presents in to our clinic with degenerative aortic valve disease with severe stenosis and mild regurgitation. Minimally invasive aortic valve replacement was performed with success in our clinic, being a safe and efficient procedure. This technique could become the standard procedure for isolated aortic valve replacement, with substantial benefits regarding respiratory function.
\end{abstract}

Keywords: aortic valve replacement, minimal invasive

\section{Introduction}

Aortic valve replacement involves median sternotomy [1]. The revolution of minimally invasive techniques from the last two decades lead to minimally invasive aortic valve replacement. Minimal "J" shaped sternotomy extended in to the 4 intercostal space provides good access to the aortic root and the possibility to connect the patient to the extracorporeal circulation machine without the use of new instruments [2]. This technique is indicated for isolated aortic valve replacement, regardless of age, particularly in patients with respiratory dysfunction because it conserves the mechanics of respiration and the pleural cavities are left intact [3]. Despite the fact that the cardiopulmonary bypass time is greater when using this technique, hospital mortality is significantly lower and five years survival is grater, when comparing with the classic technique [4]. Significant differences exist in regard with hospital stay, the need for an intra-aortic balloon contrapulsation system, reintubation and infectious complications in this patients $[5,6]$. The main disadvantages are the inability to visualize the entire heart, difficulty in deairing the heart and placing the epicardial pace weirs. The following case study describes the first minimally invasive aortic valve replacement, performed by Associate Professor Horatiu Moldovan, $\mathrm{PhD}$ and his team at the IUBC "C.C.Iliescu".

\section{Case presentation}

A 69-year-old patient presents in to our clinic with degenerative aortic valve disease with severe stenosis and mild regurgitation. The 
patient is also diagnosed with chronic obstructive lung disease stage IV Gold with secondary pulmonary hypertension, hypertension, chronic venous insufficiency, obesity and dyslipidemia. At admission the patient presents with shortness of breath, fatigue and low tolerance to exercise. Patient blood pressure is $120 / 80 \mathrm{mmHg}$ with a pulse of $80 \mathrm{bpm}$. Clinical examination reveals normal heart sounds, regular rhythm, systolic murmur grade $3 / 6$ and disseminated pulmonary crackles. Patient blood tests are in normal limits. The EKG shows normal sinus rhythm with an heart rate of 80bpm, QRS axis at 60 degrees, without other pathological signs. Transthoracic echocardiography reveals a mean transvalvular aortic gradient of $80 \mathrm{mmHg}$ with mild regurgitation, without dilatation of the aortic root. The ejection fraction is $60 \%$. The patient has normal coronary arteries evaluated by coronarography.

Considering the results of these investigations, the patient has indication for aortic valve replacement, class I, level of evidence $\mathrm{B}$. We choose the minimal invasive technique because of the associated pulmonary pathology. We further describe the operative protocol:

The patient is under general anesthesia, with central venous catheter, arterial catheter, external electrodes for defibrillation and urinary catheter placed. A minimal "J" shaped median sternotomy extended in to the 4 intercostal right space. After homeostasis control, the pericardium is incised in order to visualize the aortic root. Heparine is administrated, and the purse strings are realized at the aorta, right atrium and superior right pulmonary vein in order to vent the heart. After connection to the cardiopulmonary bypass machine and starting the bypass the aorta is clamped and cold anterograd haemopotasic cardioplegia is administered. Transverse aortotomy is realized, with a good exposure of the aortic valve, with severe calcification of the leaflets. The aortic valve is excised and a Perimount biological prosthesis is inserted using the interrupted suture technique. The aortoraphy is performed with Prolene 4.0 wires. The heart is filled with blood and deaired and the epicardial pace wires are placed on the right ventricle and right atrium. The heart is progressively disconnected from the bypass circuit and protamine is administered. The patient is stable, hemostasis is performed and pleuro-mediastinal drainage tubes are placed. In the next stage pleuro-pericadioraphy, sternoraphy with steel wires, double trim and intradermic suture are performed.

The evolution of the patient in the intensive care unit is favorable. The patient is extubated 7 hours after surgery, he becomes conscious, cooperative, without motor deficits. Patient presents acute renal dysfunction and an inflammatory syndrome with a favorable evolution during the next days. The patient is in normal sinus rhythm, with vasoconstrictor support, with a blood pressure of $80-99 \mathrm{mmHg}$ and heart rate of $80-90 \mathrm{bpm}$. The vasoconstrictor support is progressively reduced. The pleuro-mediastinal drainage tubes are removed 4 days after surgery. Patient leaves the intensive care unit 5 days after surgery, and our clinic after 1 week.

\section{Discussions}

The particularity of this case is the minimally invasive approach. The patient was selected for this type of surgery because of the associated pulmonary pathology, the advantages of these technique in conserving the normal respiratory mechanics being well known. Being the first minimally invasive aortic valve replacement, it lasted almost as the classic technique. The risks associated with a longer period of extracorporeal circulation are balanced in this case by the long time benefits in regard to respiratory function and a shorter recovery time. The time needed to perform this operative technique also depends on the patient particularities, like the thickness of the thoracic wall and the position and dimensions of the heart and ascending aorta. Selection of the patients considering this variables and realizing a standardized protocol could decrease the time necessary for this procedure.

\section{Conclusions}

Minimally invasive aortic valve replacement was performed with success in our clinic, being a safe and efficient procedure. This technique 
could become the standard procedure for isolated aortic valve replacement, with substantial benefits regarding respiratory function. Conserving the integrity of the thoracic cavity, shorter recovery time, fewer infectious complications together with reduced costs could make this technique the "gold standard" for isolated aortic valve replacement.

\section{References}

[1]Kim BS, Soltesz EG, Cohn LH. Minimally invasive approaches to aortic valve surgery: Brigham experience. Semin Thorac Cardiovasc Surg 2006;18:148-153.
[2]Svensson LG, D'Agostino RS. "J" incision minimal-access valve operations. Ann Thorac Surg 1998;66:1110-1112.

[3] Von Segesser LK, Westaby S, Pomar J, Loisance $\mathrm{D}$, Groscurth P, Turina M. Less invasive aortic valve surgery: rationale and technique. Eur J Cardiothorac Surg 1999;15:781-785.

[4]Merk DR, Lehmann S, Holzhey DM et al. Minimal invasive aortic valve replacement surgery is associated with improved survival: a propensitymatched comparison. Eur J Cardiothorac Surg 2014. [5] Shahzad G, aja Umberto Benedetto, Mohamed Amrani. Aortic valve replacement through J-shaped partial upper sternotomy. J Thorac Dis. 2013;662668

[6]Sharony R, Grossi EA, Saunders PC et al. Propensity score analysis of a six-year experience with minimally invasive isolated aortic valve replacement. J Heart Valve Dis 2004;13:887-893. 